\title{
Positive association between the ratio of triglycerides to high-density lipoprotein cholesterol and diabetes incidence in Korean adults
}

\author{
Joungyoun Kim', Sang-Jun Shin², Ye-Seul Kim ${ }^{3^{*}+}$ and Hee-Taik Kang ${ }^{3,4^{*}+}$
}

\begin{abstract}
Background: Insulin resistance is associated with the incidence of diabetes and cardiovascular diseases such as myocardial infarction. The ratio of triglycerides (TG) to high-density lipoprotein cholesterol (HDL-C) (TG/HDL-C ratio) is positively correlated with insulin resistance. This study aimed to investigate the relationship between the TG/HDL-C ratio and the incidence of diabetes in Korean adults.

Methods: This retrospective study used data from the National Health Insurance Service-National Health Screening Cohort. The TG/HDL-C ratio was divided into three tertiles, the $T_{1}, T_{2}$, and $T_{3}$ groups, based on sex. We estimated the hazard ratios (HRs) and 95\% confidence intervals (Cls) for diabetes using multivariate Cox proportional hazards regression analyses.

Results: A total of 80,693 subjects aged between 40 and 79 years were enrolled. The median follow-up period was 5.9 years. The estimated cumulative incidence of diabetes in the $T_{1}, T_{2}$, and $T_{3}$ groups was $5.94 \%, 8.23 \%$, and $13.50 \%$, respectively, in men and $4.12 \%, 4.72 \%$, and $6.85 \%$, respectively, in women. Compared to $T_{1}$, the fully adjusted HRs ( $95 \% \mathrm{Cls}$ ) of the $T_{2}$ and $T_{3}$ groups for new-onset diabetes were 1.17 (1.06-1.30) and 1.47 (1.34-1.62), respectively, in men and $1.20(1.02-1.42)$ and $1.52(1.30-1.78)$, respectively, in women.
\end{abstract}

Conclusions: Increased TG/HDL-C ratio was significantly associated with a higher risk of new-onset diabetes in both sexes.

Keywords: Cholesterol, Triglycerides, Lipoproteins, HDL, Insulin resistance, Metabolic syndrome, Cardiometabolic risk factors, Diabetes mellitus

\section{Introduction}

Diabetes mellitus (DM) is a group of metabolic disorders characterized by a chronic hyperglycemic condition resulting from defects in glucose metabolism. This non-communicable disease is one of the largest global

\footnotetext{
*Correspondence: yesul86@naver.com; kanght0818@gmail.com ${ }^{\dagger}$ Ye-Seul Kim and Hee-Taik Kang contributed equally to this work ${ }^{3}$ Department of Family Medicine, Chungbuk National University Hospital, 776 1-Soonwhan-ro, Seowon-gu, Cheongju 28644, Republic of Korea Full list of author information is available at the end of the article
}

health concerns, imposing a heavy burden on public health and socio-economic development. Patients with type 2 diabetes are at a higher risk for cardio-cerebrovascular diseases, dementia, and some malignant neoplasms than those without diabetes [1]. According to the Global Burden of Disease Study 2017, the incidence and prevalence of diabetes increased significantly from 1990 to 2017. In 2017, 22.9 million new cases and 1.37 million diabetes-related deaths globally, and 476.0 million patients continued to suffer from this disease [2]. In 
the US, patients with diabetes pay higher medical costs than those without the disease [3]. In addition to the direct medical expenditure, diabetes increases indirect costs due to increased absenteeism, lower productivity at work, disease-related disability, and premature deaths [3]. The prevalence of diabetes in Korea has risen consistently over the past seven years, from $11.8 \%$ in 2012 to $13.8 \%$ in 2018, based on data from the 2012-2018 Korean National Health and Nutrition Examination Survey [4].

Various etiologies such as age, race, geography, socioeconomic status, genetics, and health behaviors are involved in the development of diabetes [1]. In addition, impaired insulin secretion and insulin resistance may play an important role in developing the disease [5]. Thus, measurement of insulin resistance is essential to detect impaired glycemic control in the early phase of diabetes. The fasting insulin resistance index (FIRI) or homeostatic model assessment-insulin resistance (HOMA-IR) are widely used to measure insulin resistance [6]. However, using these indices is difficult in a clinical setting considering the complex calculation and low accuracy depending on the individual's adiposity [7]. Several studies have suggested that the ratio of triglycerides (TG) to HDL cholesterol (HDL-C) (TG/HDL-C ratio), which is commonly used in clinical settings, is a more appropriate and simple indicator of insulin resistance and is associated with cardiovascular risk $[8,9]$. If the TG/HDL-C ratio is well correlated with the incidence of diabetes, early detection or intervention based on this ratio can reduce the progression from normoglycemia or prediabetes to diabetes [10]. However, there is a lack of prospective evidence to investigate the association between the TG/HDL-C ratio and the development of diabetes, despite some cross-sectional studies in Korea [11].

Thus, this study aimed to investigate the incidence risk of diabetes based on the TG/HDL-C ratio in Korean adults without a history of diabetes, using data from the Korean National Health Insurance Service (NHIS)Health Screening (HEALS) cohort.

\section{Methods}

\section{Formation of cohort data and study population}

We extracted the data for this study from the Korean NHIS-HEALS cohort database. The database included 514,794 individuals, who randomly sampled $10 \%$ of the total population of 5.1 million. The individuals in this cohort had undergone the national general health screening between 2002 and 2003, were aged between 40 and 79 years in 2002, and were followed up through 2015.

The cohort data contain information, including that death, healthcare usage, and health screening. The variables from the NHIS were income-based insurance contributions (a proxy for income), demographic variables, date of death, cause of death, prescription records, and disease diagnosis codes. The variables from the health screening database were the known risk factors for diabetes, based on self-questionnaires (cigarette smoking status, alcohol drinking status, physical activity level, personal medical history, and family medical history) and bio-clinical laboratory results. This cohort was followed up from 2002 to 2015; however, lipid profile parameters such as serum TG, HDL-C, and low-density lipoprotein cholesterol (LDL-C) levels were measured from 2009; therefore, we set 2009 and 2010 as the baseline years.

Figure 1 presents the flowchart of the inclusion and exclusion criteria for this study. Of the initial 362,285 participants at baseline, 281,592 were excluded based on the following exclusion criteria: (1) participants who were aged 80 or older between 2009 and 2010 ( $n=7737$ ); (2) those who had fasting serum glucose levels $\geq 126 \mathrm{mg} /$ dL between 2009 and 2010 ( $\mathrm{n}=31,163$ ); (3) those who had serum TG levels $\geq 500 \mathrm{mg} / \mathrm{dL}$ between 2009 and 2010 ( $\mathrm{n}=3417$ ); (4) those who answered diabetes in self-reported questionnaire between 2009 and 2010 $(\mathrm{n}=30,703) ;(5)$ those who died from any cause between 2009 and 2010 ( $\mathrm{n}=1343)$; (6) those who were diagnosed with ischemic heart diseases (I20-I25), cerebrovascular diseases (I60-I69), or cancers (C00-C97) based on the 10th edition of the International Classification of Diseases (ICD-10) between 2002 and $2010(\mathrm{n}=81,235)$; (7) those who were diagnosed with diabetes (E10-E14) and prescribed any anti-diabetic drugs between 2002 and 2010 ( $\mathrm{n}=41,294)$; (8) those who were prescribed anti-dyslipidemic drugs those who were prescribed antidyslipidemic drugs (statins, ezetimibe, fibric acids, cholesterol sequestrants, and omega-3 fatty acids) between 2002 and 2015 ( $\mathrm{n}=159,567)$; (9) those who were prescribed anti-diabetic drugs without diagnosis of diabetes between 2002 and 2015 ( $\mathrm{n}=1100)$; (10) those who had incomplete data for the confounding variables $(\mathrm{n}=$ $67,400)$; or (11) those who had total study duration less or equal to 30 days $(\mathrm{n}=114)$. Participants were sequentially excluded based on the criteria mentioned above, and they were not mutually exclusive. After full exclusion, we included 80,693 participants $(47,046$ men and 33,647 women) in the final analyses.

The Institutional Review Board of Chungbuk National University approved the present study (CBNU202011-HR-0179), which adhered to the principles of the Declaration of Helsinki (1975).

The definitions of diabetes, subject groups, and study date We defined diabetes as the presence of one of the following conditions: (1) a record of diabetes diagnosis (ICD-10 code E11-14) and prescription of any anti-diabetic drug (insulin, sulfonylurea, metformin, 
NHIS-HEALS Random sample $(n=362,285)$

from health screening examiner between 2009 and 2010

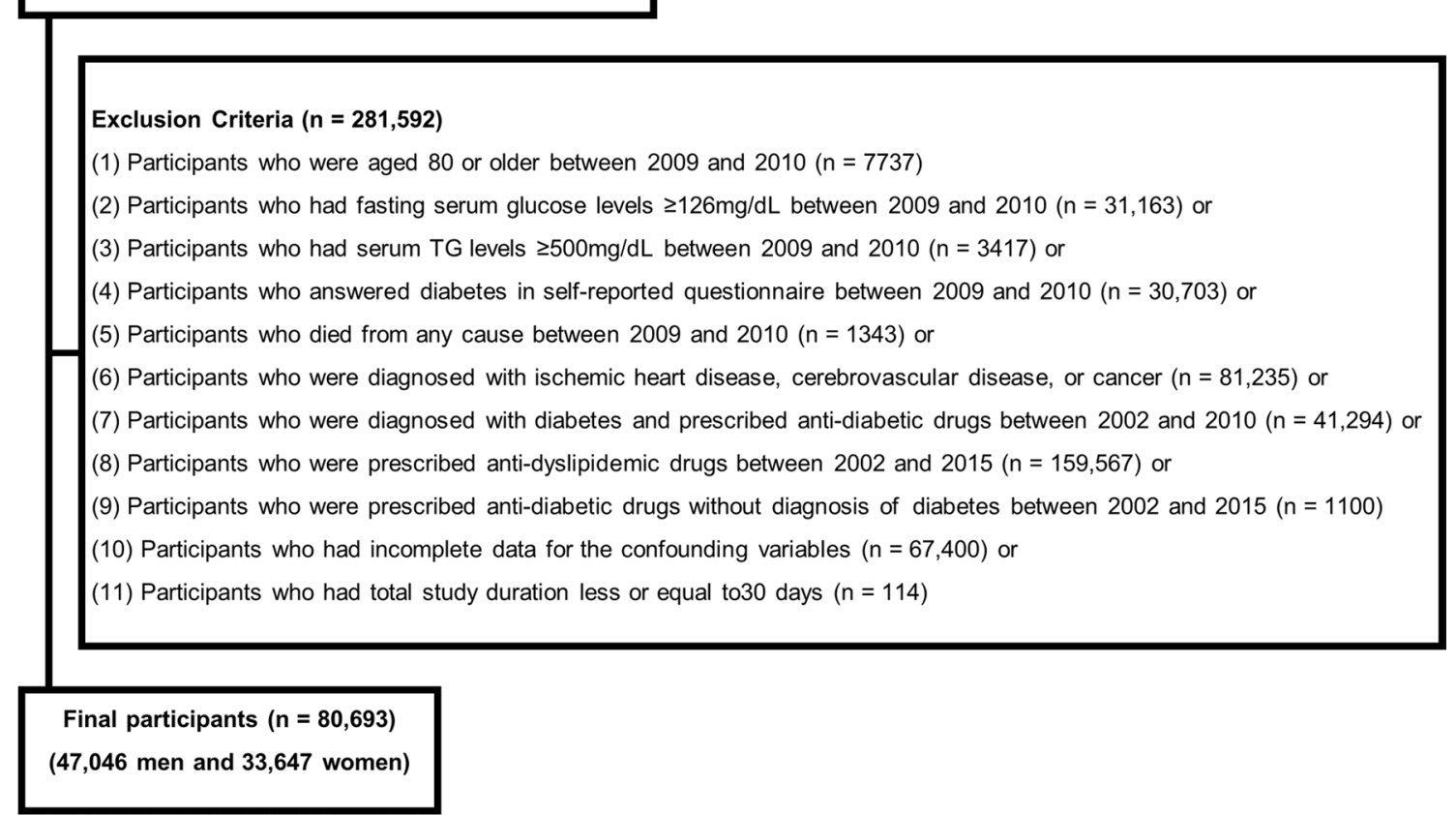

Fig. 1 Flowchart of the inclusion and exclusion criteria

thiazolidinedione, dipeptidyl peptidase-4 inhibitor, $\alpha$-glucosidase inhibitor, sodium-glucose cotransporter-2 inhibitor, glucagon-like peptide (GLP)-1 agonist, and/or others), or (2) a fasting blood glucose level $\geq 126 \mathrm{mg} / \mathrm{dL}$ on health screening. The date of incidence of diabetes was defined as the earliest between the two dates that satisfied the above conditions.

Based on analysis of the risk of diabetes according to the TG/HDL-C ratio, the participants were divided into three groups: $\mathrm{T}_{1},<1.681$ in men and $<1.278$ in women; $\mathrm{T}_{2}, 1.681-<2.951$ in men and $1.278-<2.142$ in women; and $\mathrm{T}_{3}, \geq 2.951$ in men and $\geq 2.142$ in women.

The research start date was defined as the day of the first health examination between 2009 and 2010. For the participants diagnosed with diabetes between 2011 and 2015, the research end date was the initial diagnosis date of the disease. In cases where the participant died before a diagnosis of diabetes, the end date was defined as the date of death. Similarly, in cases where the participants had not died or had not been diagnosed with diabetes during the study period, the end date was the latest date of the last outpatient clinic visit, last health screening, or last when the prescribed medication was taken.

\section{Definition of covariates}

In this study, analyses were conducted by controlling for confounding variables such as age, systolic blood pressure (SBP), body mass index (BMI) $\left(\mathrm{kg} / \mathrm{m}^{2}\right)$, serum fasting glucose, total cholesterol, and alanine aminotransferase (ALT) levels, personal history of hypertension, family history of diabetes, smoking status, alcohol consumption, physical activity, and household income levels between 2009 and 2010.

BMI was defined as body mass in kilograms divided by the square of height in meters. We collected data regarding hypertension, family history of diabetes, smoking status, alcohol consumption, and physical activity from self-reported questionnaires. Smoking status was categorized as non-smoker or ever-smoker. Non-smokers were defined as individuals who answered "No" to "Have you ever smoked 5 packs (100 cigarettes) or more in your lifetime?" in the self-reported questionnaires. Ever-smokers were defined as individuals who answered "Yes" to this question [12,13]. Alcohol consumption was classified as rare (less than once a week), sometimes (once to twice per week), and often (three or more times per week) [14]. Physical activity was divided into three categories: rare (less than once a week of any intensity exercise), sometimes (not meeting the definition of the rare or regular categories), and regular (five or more times of walking 
or moderate-intensity exercise per week; three or more times of vigorous-intensity exercise per week; four or more times of walking [or moderate-intensity exercise] and vigorous-intensity exercise per week) [15]. We categorized monthly household income into three groups: low, 0-3rd deciles; middle, 4th-7th deciles; and high, 8th-10th deciles.

\section{Statistical analysis}

Continuous variables are expressed as mean \pm standard deviation (SD). Categorical variables are expressed as the number of subjects (percentage). For group comparison, analysis of variance (ANOVA) and Chi-square tests were used for the continuous and categorical variables, respectively.

To determine the association between the TG/HDL-C ratio and the incidence of diabetes, we estimated and compared diabetes-free survival rates using the KaplanMeier method based on the log-rank test. Cox proportional hazards regression models were adopted to calculate the hazard ratios (HRs) and 95\% confidence intervals (95\% CIs) after controlling for confounding factors. Four Cox proportional hazards regression models were considered: (1) Model 1: age only; (2) Model 2: age, smoking status, alcohol consumption, and physical activity; (3) Model 3: BMI, SBP, total cholesterol level, ALT level, history of hypertension, family history of diabetes, and household income in addition to the variables in Model 2; and (4) Model 4: fasting glucose level in addition to the variables in Model 3.

All p-values reported are two-sided, and statistical significance was set at $\mathrm{p}<0.05$. The statistical packages SAS enterprise guide version 7.1 (SAS Inc., Cary, NC) and $\mathrm{R}$ studio version 3.3.3 were used to perform the study analyses.

\section{Results}

The total study population was 80,693 (47,046 men and 33,647 women), and the median follow-up duration was 5.9 years. Table 1 shows the baseline characteristics of the study population based on the TG/HDL-C ratio. BMI, SBP, serum fasting glucose, total cholesterol, ALT levels, and percentage of hypertension increased the higher TG/ HDL-C ratio in both sexes (Table 1). The higher tertile groups of the TG/HDL-C ratio had fewer regular alcohol drinkers and a less physically active population than in the lower tertile group (all p value $<0.001$ ). Men in the higher tertile groups were younger, while women were older. Household income, percentage of a family history of diabetes, and the number of ever-smokers were higher in the higher tertile groups than in the lower tertile group among men, while the variables were not statistically significant in women.
Figure 2 shows the estimated cumulative incidence rates of diabetes based on the Kaplan-Meier survival curve. Cumulative incidence was the highest in $\mathrm{T}_{3}$ and lowest in $T_{1}$ in both sexes (log-rank test $\mathrm{p}$ values < 0.001). A total of 4093 cases of diabetes were observed, accounting for $5.1 \%$ of the total participants. At the end of the follow-up period, the estimated cumulative incidences of diabetes from $\mathrm{T}_{1}$ to $\mathrm{T}_{3}$ were $5.94 \%, 8.23 \%$, and $13.50 \%$, respectively, in men and $4.12 \%, 4.72 \%$, and $6.85 \%$, respectively, in women.

Table 2 shows the results of the Cox proportional hazards regression models (Table 2). We used Cox proportional hazards regression models to quantify the risk of diabetes according to the TG/HDL-C ratio. Compared with $\mathrm{T}_{1}$, HRs (95\% CIs) for the incidence of diabetes in $\mathrm{T}_{2}$ and $\mathrm{T}_{3}$ were $1.35(1.23-1.49)$ and 1.93 (1.76-2.12), respectively, in men and 1.39 (1.19-1.64) and 2.02 (1.73$2.35)$, respectively, in women after adjusting for age only (Model 1). After adjusting for the variables included in Model 3, HRs (95\% CIs) for diabetes incidence of $\mathrm{T}_{2}$ and $\mathrm{T}_{3}$ were $1.22(1.10-1.34)$ and 1.57 (1.42-1.73), respectively, in men and 1.27 (1.08-1.49) and 1.64 (1.41-1.92), respectively, in women (Model 3). Furthermore, we adjusted fasting glucose levels to minimize the effects of baseline glucose levels in Model 4. After full adjustment, the HRs (95\% CIs) for the incidence of diabetes in $\mathrm{T}_{2}$ and $\mathrm{T}_{3}$ were 1.17 (1.06-1.30) and 1.47 (1.34-1.62), respectively, in men and $1.20(1.02-1.42)$ and $1.52(1.30-1.78)$, respectively, in women (Model 4).

We examined the area under the curves (AUCs) of fasting glucose, TG/HDL ratio, and the combination of TG/ HDL-C ratio and fasting glucose (Additional file 1: Figure S1). In all models, fasting glucose had a better predictive power than the TG/HDL-C ratio. The AUCs of the receiver operating curve (ROC) analyses were 0.708 , $0.604,0.718$, respectively, in all participants; $0.686,0.586$, and 0.695 , respectively, in men; and 0.732, 0.596, and 0.738 , respectively, in women. Delong's test was performed for all possible pairwise comparisons to test for significant differences between the ROC curves. In each comparison, the $\mathrm{p}$ value was less than 0.001 .

\section{Discussion}

This retrospective study demonstrated that an elevated TG/HDL-C ratio was positively associated with the incidence of diabetes in the Korean population, based on the Korean NHIS-HEALS data. Our study provides additional information that high TG and low HDL-C levels, as surrogate markers of insulin resistance, may predict the risk of diabetes in primary clinical settings.

Insulin resistance is a crucial pathophysiological parameter of metabolic syndrome, which is a cluster of metabolic risk factors associated with an increased 
Table 1 Baseline characteristics of the study participants according to the TG/HDL-C ratio groups

\begin{tabular}{|c|c|c|c|c|}
\hline Men & $\mathrm{T}_{1}(<1.681)$ & $\mathrm{T}_{2}(1.681-<2.951)$ & $\mathrm{T}_{3}(\geq 2.951)$ & p value \\
\hline Number of subjects & 15,692 & 15,675 & 15,679 & NA \\
\hline $\mathrm{TG} / \mathrm{HDL}-\mathrm{C}$ & $1.17 \pm 0.32$ & $2.25 \pm 0.36$ & $4.84 \pm 1.99$ & $<0.001$ \\
\hline Age, yr & $56.5 \pm 7.8$ & $55.8 \pm 7.5$ & $55.0 \pm 7.1$ & $<0.001$ \\
\hline $\mathrm{BMI}, \mathrm{kg} / \mathrm{m}^{2}$ & $22.7 \pm 2.6$ & $23.8 \pm 2.6$ & $24.6 \pm 2.5$ & $<0.001$ \\
\hline $\mathrm{SBP}, \mathrm{mmHg}$ & $122.9 \pm 14.5$ & $124.6 \pm 14.3$ & $126.0 \pm 14.2$ & $<0.001$ \\
\hline Fasting glucose, $\mathrm{mg} / \mathrm{dL}$ & $94.2 \pm 11.1$ & $95.4 \pm 11.2$ & $96.4 \pm 11.5$ & $<0.001$ \\
\hline Total cholesterol, mg/dL & $186.6 \pm 29.4$ & $191.4 \pm 30.7$ & $195.3 \pm 31.8$ & $<0.001$ \\
\hline $\mathrm{TG}, \mathrm{mg} / \mathrm{dL}$ & $71.3 \pm 22.4$ & $115.2 \pm 24.9$ & $205.6 \pm 71.2$ & $<0.001$ \\
\hline $\mathrm{HDL}-\mathrm{C}, \mathrm{mg} / \mathrm{dL}$ & $64.4 \pm 38.8$ & $51.4 \pm 9.3$ & $43.8 \pm 8.6$ & $<0.001$ \\
\hline ALT, IU/L & $23.4 \pm 17.4$ & $25.4 \pm 16.5$ & $29.2 \pm 19.9$ & $<0.001$ \\
\hline Hypertension, N (\%) & $2086(13.2)$ & $2534(16.2)$ & $2618(16.7)$ & $<0.001$ \\
\hline Family history of diabetes, N (\%) & $832(5.3)$ & $906(5.8)$ & $1036(6.6)$ & $<0.001$ \\
\hline Ever-smokers, N (\%) & $9308(59.3)$ & $9981(63.7)$ & $10,728(68.4)$ & $<0.001$ \\
\hline Alcohol consumption, N (\%) & & & & $<0.001$ \\
\hline Rare & $5450(34.7)$ & $5584(35.6)$ & $5318(33.9)$ & \\
\hline Sometimes & $6500(41.4)$ & $6553(41.8)$ & $6799(43.4)$ & \\
\hline Regular & $3742(23.8)$ & $3538(22.6)$ & $3562(22.7)$ & \\
\hline Physical activity, N (\%) & & & & $<0.001$ \\
\hline Rare & $3288(21.0)$ & $3186(20.3)$ & $3302(21.1)$ & \\
\hline Sometimes & $6903(44.0)$ & $7513(47.9)$ & 7751 (49.4) & \\
\hline Regular & $5501(35.1)$ & $4976(31.7)$ & $4626(29.5)$ & \\
\hline Household income, N (\%) & & & & $<0.001$ \\
\hline Low & $2301(14.7)$ & $2224(14.2)$ & $2064(13.2)$ & \\
\hline Middle & $4790(30.5)$ & $4609(29.4)$ & $4585(29.2)$ & \\
\hline High & $8601(54.8)$ & $8842(56.4)$ & $9030(57.6)$ & \\
\hline Women & $\mathrm{T}_{1}(<1.278)$ & $\mathrm{T}_{2}(1.278-<2.142)$ & $\mathrm{T}_{3}(\geq 2.142)$ & $\mathrm{p}$ value \\
\hline Number of subjects & 11,227 & 11,207 & 11,213 & NA \\
\hline TG/HDL-C & $0.92 \pm 0.24$ & $1.67 \pm 0.25$ & $3.54 \pm 1.58$ & $<0.001$ \\
\hline Age, yr & $54.5 \pm 6.8$ & $56.3 \pm 7.7$ & $58.3 \pm 8.4$ & $<0.001$ \\
\hline $\mathrm{BMI}, \mathrm{kg} / \mathrm{m}^{2}$ & $22.7 \pm 2.7$ & $23.4 \pm 2.9$ & $24.1 \pm 2.9$ & $<0.001$ \\
\hline $\mathrm{SBP}, \mathrm{mmHg}$ & $118.2 \pm 14.6$ & $120.6 \pm 15.1$ & $123.6 \pm 15.6$ & $<0.001$ \\
\hline Fasting glucose, mg/dL & $91.1 \pm 10.0$ & $92.2 \pm 10.1$ & $93.2 \pm 10.5$ & $<0.001$ \\
\hline Total cholesterol, mg/dL & $193.2 \pm 29.9$ & $194.9 \pm 30.2$ & $198.8 \pm 31.3$ & $<0.001$ \\
\hline $\mathrm{TG}, \mathrm{mg} / \mathrm{dL}$ & $60.9 \pm 19.8$ & $94.3 \pm 19.1$ & $163.5 \pm 58.4$ & $<0.001$ \\
\hline $\mathrm{HDL}-\mathrm{C}, \mathrm{mg} / \mathrm{dL}$ & $70.8 \pm 47.7$ & $56.8 \pm 9.7$ & $47.9 \pm 9.1$ & $<0.001$ \\
\hline$A L T, I U / L$ & $19.4 \pm 19.7$ & $20.2 \pm 14.8$ & $21.9 \pm 14.8$ & $<0.001$ \\
\hline Hypertension, N (\%) & $1288(11.5)$ & $1769(15.8)$ & $2299(20.5)$ & $<0.001$ \\
\hline Family history of diabetes, N (\%) & $912(8.1)$ & $861(7.7)$ & $815(7.3)$ & 0.056 \\
\hline Ever-smokers, N (\%) & $194(1.7)$ & $207(1.8)$ & $238(2.1)$ & 0.085 \\
\hline Alcohol consumption, N (\%) & & & & $<0.001$ \\
\hline Rare & $8979(80.0)$ & $9484(84.6)$ & $9736(86.8)$ & \\
\hline Sometimes & $1905(17.0)$ & $1429(12.8)$ & $1255(11.2)$ & \\
\hline Regular & $343(3.1)$ & $294(2.6)$ & $222(2.0)$ & \\
\hline Physical activity, N (\%) & & & & $<0.001$ \\
\hline Rare & $3020(26.9)$ & $3382(30.2)$ & $3602(32.1)$ & \\
\hline Sometimes & $4914(43.8)$ & $4736(42.3)$ & $4723(42.1)$ & \\
\hline Regular & $3293(29.3)$ & $3089(27.6)$ & $2888(25.8)$ & \\
\hline Household income, N (\%) & & & & 0.080 \\
\hline Low & $2906(25.9)$ & $2839(25.3)$ & $2797(24.9)$ & \\
\hline Middle & $3925(35.0)$ & $4021(35.9)$ & $4125(36.8)$ & \\
\hline High & $4396(39.2)$ & $4347(38.8)$ & 4291 (38.3) & \\
\hline
\end{tabular}


Table 1 (continued)

Values are presented as $\mathrm{n}(\%)$ or mean \pm standard deviation

SBP systolic blood pressure; BMI body mass index; TG triglyceride; HDL-C HDL cholesterol; TG/HDL-C ratio the ratio of TG to HDL-C; ALT alanine aminotransferase

(a) Men

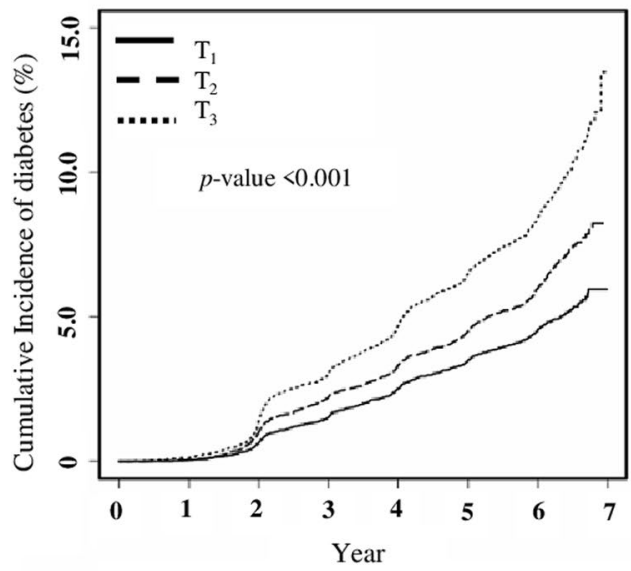

$\begin{array}{lllllllll}\mathrm{T}_{1} & 0 & 0.05 & 0.65 & 1.53 & 2.50 & 3.47 & 4.55 & 5.94\end{array}$

$\begin{array}{lllllllll}\mathrm{T}_{2} & 0 & 0.05 & 1.03 & 2.21 & 3.31 & 4.49 & 5.98 & 8.23\end{array}$

$\begin{array}{lllllllll}\mathrm{T}_{3} & 0 & 0.13 & 1.45 & 3.07 & 4.69 & 6.51 & 8.43 & 13.50\end{array}$

(b) Women

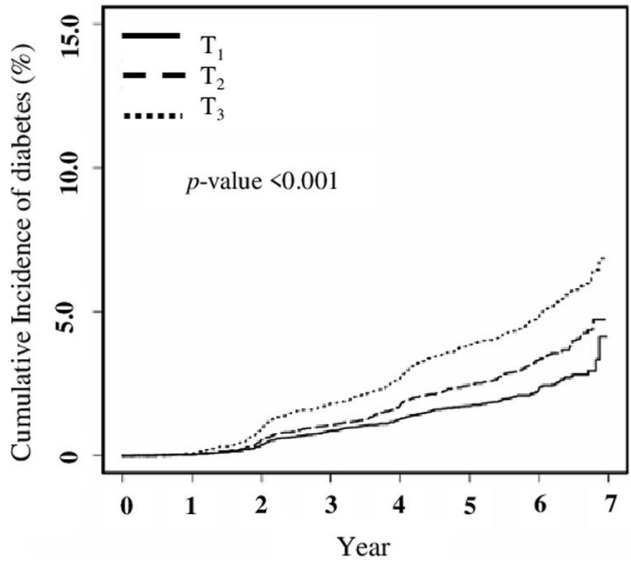

$\begin{array}{lllllllll}\mathrm{T}_{1} & 0 & 0.05 & 0.38 & 0.86 & 1.27 & 1.71 & 2.34 & 4.12\end{array}$

$\begin{array}{llllllllll}\mathrm{T}_{2} & 0 & 0.03 & 0.55 & 1.05 & 1.73 & 2.43 & 3.34 & 4.72\end{array}$

$\begin{array}{lllllllll}\mathrm{T}_{3} & 0 & 0.09 & 0.92 & 1.80 & 2.67 & 3.86 & 4.84 & 6.85\end{array}$

* Cumulative incidence is 1 -survival rate. p-value is from the log-rank test.

Fig. 2 The estimated cumulative incidence of type 2 diabetes mellitus

Table 2 Cox proportional hazards regression results for diabetes incidence according to the TG/HDL-C ratio groups

\begin{tabular}{|c|c|c|c|c|c|c|}
\hline \multirow[t]{2}{*}{ HRs (95\% Cls) } & \multicolumn{3}{|l|}{ Men } & \multicolumn{3}{|l|}{ Women } \\
\hline & $\mathrm{T}_{1}(<1.681)$ & $\mathrm{T}_{2}(1.681-<2.951)$ & $\mathrm{T}_{3}(\geq 2.951)$ & $\mathrm{T}_{1}(<1.278)$ & $\mathrm{T}_{2}(1.278-<2.142)$ & $\mathrm{T}_{3}(\geq 2.142)$ \\
\hline Model 1 & 1 & $1.35(1.23-1.49)$ & $1.93(1.76-2.12)$ & 1 & $1.39(1.19-1.64)$ & $2.02(1.73-2.35)$ \\
\hline Model 2 & 1 & $1.36(1.23-1.50)$ & $1.93(1.78-2.12)$ & 1 & $1.40(1.19-1.65)$ & $2.04(1.75-2.37)$ \\
\hline Model 3 & 1 & $1.22(1.10-1.34)$ & $1.57(1.42-1.73)$ & 1 & $1.27(1.08-1.49)$ & $1.64(1.41-1.92)$ \\
\hline Model 4 & 1 & $1.17(1.06-1.30)$ & $1.47(1.34-1.62)$ & 1 & $1.20(1.02-1.42)$ & $1.52(1.30-1.78)$ \\
\hline
\end{tabular}

Model 1: adjusted for age

Model 2: adjusted for smoking status, alcohol consumption, and physical activity in addition to Model 1

Model 3: adjusted for body mass index, systolic blood pressure, serum total cholesterol, alanine aminotransferase, past hypertension history, family history of diabetes, and monthly household income, in addition to Model 2

Model 4: adjusted for fasting glucose levels, in addition to Model 3

risk for type 2 diabetes and cardiovascular diseases [16]. Insulin resistance means that insulin stimuli fail to induce an appropriate biologic response, such as glycemic control. Consequently, the human body increases the production of pancreatic insulin and results in hyperinsulinemia to compensate for the dysfunction. The exact measurement of insulin resistance preceding type 2 diabetes is very important to prevent its harmful consequences, including diabetes and cardiovascular diseases $[17,18]$. Several methods have been suggested to measure insulin resistance. The hyperinsulinemiceuglycemic glucose clamp technique is considered the gold standard method to assess insulin resistance. However, it is not easy to apply this technique in real-world clinical settings considering the complex measurement procedure [19]. HOMA-IR is commonly used to assess 
insulin resistance [20]; however, it has limited applicability. Its calculation involves a complex equation and measured fasting insulin levels. The TG/HDL-C ratio is another reliable surrogate marker to assess insulin resistance [21, 22]. Lipid profile parameters such as TG and HDL-C are inexpensive laboratory tests; therefore, they are more commonly used to assess cardiovascular risk than insulin. In addition, the TG/HDL-C ratio can be calculated easily by dividing TG with HDL-C. A cross-sectional study reported the correlation between the TG/HDL-C ratio and insulin resistance regardless of waist circumference in the Korean adult population [11]. Although our results are consistent with several previous studies in that the TG/HDL-C ratio was significantly associated with cardiovascular risk factors and the incidence of diabetes [23-25], there remain contradictory claims that the ratio is not a reliable indicator of insulin resistance. Thus, further research is needed [26].

The mechanism by which a high TG/HDL-C ratio increases the incidence of diabetes is unclear, although the ratio appears to be a relatively reliable indicator of insulin resistance. Previous studies have suggested dyslipidemia as a causal factor of insulin resistance [27]. An increase in TG and decreased HDL-C levels through genetic variants in lipid-related genes could cause insulin resistance [28]. It results in compensatory hyperinsulinemia, leading to aggravation of hypertriglyceridemia. Other studies have reported that lipotoxicity might play a role in insulin resistance and pancreatic $\beta$-cell dysfunction by producing inflammatory cytokines, mitochondrial oxidation, and the protein kinase $\mathrm{C}$ and c-Jun NH2-terminal kinase (JNK)-1 pathways in non-adipose tissue organs [29-31]. In addition, it has been suggested that hypertriglyceridemia and insulin resistance are linked through the cholesteryl ester transfer protein (CETP). The CETP is a glycoprotein that regulates the equilibrium of lipoprotein fractions by promoting the bidirectional transfer of cholesteryl esters (CE) and TG [32]. In previous studies on hypertriglyceridemia patients, CETP activity was elevated. This elevated activity caused the conversion of TG to TG-enriched LDL and HDL to small-dense HDL, resulting in decreased HDL-C levels [33, 34]. Although the mechanism of the association between CETP and insulin resistance has not yet been precisely elucidated, previous studies showed that when a CETP inhibitor was used, HDL-C increased, and the HOMA-IR and glycemic control in type 2 diabetic patients were improved $[35,36]$. This study explained that the CETP inhibitor possibly increased the insulinstimulated glucose uptake in the skeletal muscles, liver, and heart, improving glucose homeostasis [35]. These results implied the possibility that CETP may also affect glucose metabolism and insulin action regulation in addition to its action in lipoprotein metabolism.

Insulin resistance tends to increase with age, obesity, and an unhealthy lifestyle, such as limited physical activity [37-39]. This trend was expected to be observed in the TG/HDL-C ratio as well, which indirectly reflects insulin resistance. Contrary to expectations, in our study, the men in the group with a higher TG/HDL-C ratio were younger and had higher household incomes. The group with a lower TG/HDL-C ratio had a higher percentage of participants (both men and women) who consumed alcohol regularly. Additional adjustment for fasting blood glucose levels at baseline was performed. Fasting blood glucose levels are one of the most critical risk factors for predicting the development of diabetes. Even after fully adjustment, the TG/HDL-C ratio was significantly associated with the incidence of diabetes in both men and women. This result supports the findings reported by Qi et al. [40] in that the TG/HDL-C ratio can increase the risk of type 2 diabetes independent of insulin resistance, similar to genetic predisposition.

Our study has several limitations. First, because the NHIS-HEALS cohort did not collect serum insulin levels, we could not validate the correlation between the TG/HDL-C ratio and insulin resistance. By validating this correlation, it can be concluded that the positive association between the TG/HDL-C ratio and diabetes risk results from insulin resistance. However, increased TG/ HDL-C ratio may influence the occurrence of diabetes through its unique properties such as genetic predisposition, apart from insulin resistance. Second, the type of diabetes, the diagnosis time, and the incidence may differ from the actual rate due to the limitations of cohort data. Although this study tried to confirm type $2 \mathrm{DM}$, the incidence of diabetes was defined based on the fasting glucose level during the biannual health screening tests or the prescription of anti-diabetic medications combined with clinical diagnostic codes of diabetes-related diseases. Diabetes was diagnosed based on the following criteria: (1) fasting blood glucose $\geq 126 \mathrm{mg} / \mathrm{dL}$ after at least 8-h fasting; (2) glycated hemoglobin (HbA1C $\geq 6.5 \%$ ( $48 \mathrm{mmol} / \mathrm{mol}$ ); 2 -h blood glucose $\geq 200 \mathrm{mg} / \mathrm{dL}$ during an oral glucose tolerance test; and random blood glucose $\geq 200 \mathrm{mg} / \mathrm{dL}$ with classic hyperglycemic symptoms [41]. This operational definition of diabetes adopted in this study might have led to inaccurate results. In addition, the actual diagnosis or onset of diabetes could be different from the real-world data. However, the incidence rate of type $1 \mathrm{DM}$ in Korean adults over 20 years of age is 2.99 per 1,00,000 [42]. As such, the possibility of type $1 \mathrm{DM}$ occurrence in this study is considered low.

Third, we could not include other risk factors for diabetes due to limited data availability. In particular, dietary 
patterns in which foods with a high glycemic index or load are consumed can increase the risk of diabetes [43, 44]. Unfortunately, the NHIS-HEALS cohort did not include this information. Fourth, there is no consensus regarding the normal range or cut-off value of the TG/ HDL-C ratio. Previous studies have shown that the TG/ HDL-C ratio value differed according to age, sex, diet, country, race, and culture $[45,46]$. Wakabayashi et al's studies reported TG/HDL-C ratio varied age and sex and showed a J-shaped relationship with alcohol intake in a hypertensive population [47, 48]. Another study reported that diet significantly altered the lipid profile [49]. Also, since this study demonstrated a positive correlation in a dose-dependent manner, we believe that the TG/HDL-C ratio is a risk factor for diabetes. In addition, the TG/ HDL-C ratio can be easily measured in primary clinical settings and can provide additional information regarding cardiovascular diseases.

Despite several limitations, this study has certain advantages over other studies. First, the Korean NHISHEALS cohort represents the entire Korean population. A retrospective cohort study was recently published to present the association between the TG/HDL-C ratio and the risk of diabetes [50]. However, the study as mentioned earlier, might not represent the characteristics of the general Korean population because it analyzed a cohort consisting of residents in two regions. In contrast, since our study included a sample of NHIS-insured individuals, the results can be generalized to a significantly larger population. Second, we adopted a relatively conservative approach to minimize unexpected biases. Individuals who had taken any lipid-lowering drugs, such as statins, ezetimibe, fibric acid, cholesterol sequestrants, and omega- 3 fatty acids, were excluded by using the ATC code from this study. For instance, most statins that reduce cholesterol levels and prevent cardiovascular diseases can increase blood glucose levels and cause newonset diabetes [51]. Previous studies have reported that statin treatment affects the calcium channel of the pancreatic $\beta$-cells by altering insulin secretion or decreasing glucose transporter 4 translocation, leading to hyperglycemia and hyperinsulinemia, which can be related to the development of diabetes [52]. And, omega-3 fatty acids could affect the study results because they lower the serum TG level by increasing $\beta$-oxidation and, decreasing the hepatic fatty acids synthesis $[53,54]$. Third, the median follow-up duration of 5.9 years is relatively long. Chronic diseases, such as diabetes, require a long time from exposure to clinical onset. Therefore, a sufficient follow-up period is essential to analyze the association in a favorable manner.

The normal range or cut-off value of the TG/HDL-C ratio for increased risk of diabetes in healthy adults or diabetic risk groups has not been standardized. Through the ROC analysis results in this study, the predictive power of diabetes using the TG/HDL-C ratio alone was lower than that of the fasting glucose. However, compared to the model using fasting glucose alone, the model's predictive power improved after combining the TG/ HDL-C ratio and fasting glucose. So, the TG/HDL-C ratio, a relatively simple test to perform in the clinic, can be used as an additional diagnostic tool. Assessment of this ratio might help identify the risk group for diabetes transition and plan lifestyle modifications.

In conclusion, elevated TG/HDL-C ratio was positively associated with increased diabetes incidence in the Korean population.

\section{Abbreviations}

ALT: Alanine aminotransferase; ANOVA: Analysis of variance; AUC: Area under the curves; BMI: Body mass index; CE: Cholesteryl esters; CETP: Cholesteryl ester transfer protein; CI: Confidence interval; DM: Diabetes mellitus; FIRI: Fasting insulin resistance index; GLP: Glucagon-like peptide; HDL-C: High-density lipoprotein cholesterol; HR: Hazard ratio; HOMA-IR: Homeostatic model assessment-insulin resistance; HEALS: Health screening; ICD: International classification of diseases; LDL-C: Low-density lipoprotein cholesterol; NHIS: National Health Insurance Service; ROC: Receive operator curve; SBP: Systolic blood pressure; SD: Standard deviation; SNP: Single nucleotide polymorphism; TG: Triglycerides; TG/HDL-C ratio: The ratio of triglyceride to high-density lipoprotein cholesterol.

\section{Supplementary Information}

The online version contains supplementary material available at https://doi. org/10.1186/s12933-021-01377-5.

Additional file 1: Figure S1. ROC curve analysis of TG/HDL-C ratio and fasting glucose as a predictor of DM incidence.

\section{Acknowledgements}

This work was supported by the National Research Foundation of Korea (NRF) Grant funded by the Korean government (MSIT) (Grant No. 2021R1G1A1006485 and 2019111A3A01059886).

\section{Authors' contributions}

All authors made substantial contributions to the conception or design of the work. All the authors made substantial contributions to the acquisition, analysis, or interpretation of data for the work. All authors contributed to the manuscript drafting or critically revised it for important intellectual content and reviewed and approved the final manuscript. Y-SK and H-TK are the guarantors of this work and, as such, had full access to all data in the study and takes responsibility for the integrity of the data and the accuracy of the data analysis. All authors read and approved the final manuscript.

\section{Funding}

Joungyoun Kim received a research grant by the National Research Foundation of Korea (NRF) Grant Funded by the Korea government (MSIT) (Grant Number: 2019R1I1A3A01059886). Hee-Taik Kang received a grant of the Korea Health Technology R\&D Project through the Korea Health Industry Development Institute (KHIDI), Funded by the Ministry of Health \& Welfare, Republic of Korea (Grant Number: HI19C0526) and a research grant by the National Research Foundation of Korea (NRF) Grant Funded by the Korea government (Grant Number: 2021R1G1A1006485).

Availability of data and materials Not applicable. 


\section{Declarations}

Ethics approval and consent to participate

Not applicable.

\section{Consent for publication}

Not applicable.

\section{Competing interests}

The authors declare that they have no competing interests.

\section{Author details}

${ }^{1}$ College of Nursing, Mo-Im Kim Nursing Research Institute, Yonsei University, 50-1, Yonsei-Ro, Seodaemun-gu, Seoul 03722, Republic of Korea. ${ }^{2}$ Department of Information and Statistics, Chungbuk National University, 1 Chungdae-ro, Seowon-gu, Cheongju, Chungbuk 28644, Republic of Korea. ${ }^{3}$ Department of Family Medicine, Chungbuk National University Hospital, 776 1-Soonwhan-ro, Seowon-gu, Cheongju 28644, Republic of Korea. ${ }^{4}$ Department of Family Medicine, Chungbuk National University College of Medicine, 1 Chungdae-ro, Seowon-gu, Cheongju, Chungbuk 28644, Republic of Korea.

Received: 13 June 2021 Accepted: 2 September 2021

Published online: 09 September 2021

\section{References}

1. Skyler JS, Bakris GL, Bonifacio E, Darsow T, Eckel RH, Groop L, Groop P-H, Handelsman Y, Insel RA, Mathieu C. Differentiation of diabetes by pathophysiology, natural history, and prognosis. Diabetes. 2017;66(2):241-55.

2. Lin X, Xu Y, Pan X, Xu J, Ding Y, Sun X, Song X, Ren Y, Shan P-F. Global, regional, and national burden and trend of diabetes in 195 countries and territories: an analysis from 1990 to 2025. Sci Rep. 2020;10(1):1-11.

3. Association AD. Economic costs of diabetes in the US in 2017. Diabetes Care. 2018:41(5):917-28.

4. Kim B-Y, Won JC, Lee JH, Kim H-S, Park JH, Ha KH, Won KC, Kim DJ, Park KS. Diabetes Fact Sheets in Korea, 2018: an appraisal of current status. Diabetes Metab J. 2019;43(4):487-94.

5. Ozougwu J, Obimba K, Belonwu C, Unakalamba C. The pathogenesis and pathophysiology of type 1 and type 2 diabetes mellitus. J Physiol Pathophysiol. 2013;4(4):46-57.

6. Duncan M, Singh B, Wise PH, Carter G, Alaghband-Zadeh J. A simple measure of insulin resistance. The Lancet. 1995;346(8967):120-1.

7. Seo YG, Song HJ, Song YR. Fat-to-muscle ratio as a predictor of insulin resistance and metabolic syndrome in Korean adults. J Cachexia Sarcopenia Muscle. 2020. https://doi.org/10.1002/jcsm.12548.

8. Lin D, Qi Y, Huang C, Wu M, Wang C, Li F, Yang C, Yan L, Ren M, Sun K. Associations of lipid parameters with insulin resistance and diabetes: a population-based study. Clin Nutr. 2018;37(4):1423-9.

9. Murguía-Romero M, Jiménez-Flores JR, Sigrist-Flores SC, Espinoza-Camacho MA, Jiménez-Morales M, Piña E, Méndez-Cruz AR, Villalobos-Molina R, Reaven GM. Plasma triglyceride/HDL-cholesterol ratio, insulin resistance, and cardiometabolic risk in young adults. J Lipid Res. 2013;54(10):2795-9.

10. Phillips LS, Ratner RE, Buse JB, Kahn SE. We can change the natural history of type 2 diabetes. Diabetes Care. 2014;37(10):2668-76.

11. Kang H-T, Yoon J-H, Kim J-Y, Ahn S-K, Linton J, Koh S-B, Kim J-K. The association between the ratio of triglyceride to $\mathrm{HDL}-\mathrm{C}$ and insulin resistance according to waist circumference in a rural Korean population. Nutr Metab Cardiovasc Dis. 2012;22(12):1054-60.

12. Seong SC, Kim Y-Y, Park SK, Khang YH, Kim HC, Park JH, Kang H-J, Do C-H, Song J-S, Lee E-J. Cohort profile: the national health insurance servicenational health screening cohort (NHIS-HEALS) in Korea. BMJ Open. 2017;7(9):e016640.

13. Pomerleau CS, Pomerleau OF, Snedecor SM, Mehringer AM. Defining a never-smoker: results from the nonsmokers survey. Addict Behav. 2004;29(6):1149-54

14. Health Do. UK Chief Medical Officers' low risk drinking guidelines. 2016. https://assets.publishing.service.gov.uk/government/uploads/system/ uploads/attachment_data/file/545937/UK_CMOs_report.pdf.
15. Piercy KL, Troiano RP, Ballard RM, Carlson SA, Fulton JE, Galuska DA, George SM, Olson RD. The physical activity guidelines for Americans. JAMA. 2018:320(19):2020-8.

16. Saely $\mathrm{CH}$, Aczel S, Marte T, Langer P, Hoefle G, Drexel $H$. The metabolic syndrome, insulin resistance, and cardiovascular risk in diabetic and nondiabetic patients. J Clin Endocrinol Metab. 2005;90(10):5698-703.

17. Ormazabal V, Nair S, Elfeky O, Aguayo C, Salomon C, Zuñiga FA. Association between insulin resistance and the development of cardiovascular disease. Cardiovasc Diabetol. 2018;17(1):1-14.

18. Kahn $\mathrm{S}$. The relative contributions of insulin resistance and beta-cell dysfunction to the pathophysiology of type 2 diabetes. Diabetologia. 2003:46(1):3-19.

19. DeFronzo RA, Tobin JD, Andres R. Glucose clamp technique: a method for quantifying insulin secretion and resistance. Am J Physiol Endocrinol Metab. 1979;237(3):E214.

20. Fukushima M, Taniguchi A, Sakai M, Doi K, Nagasaka S, Tanaka H, Tokuyama K, Nakai Y. Homeostasis model assessment as a clinical index of insulin resistance. Diabetes Care. 1999:22(11):1911-1911.

21. Singh B, Saxena A. Surrogate markers of insulin resistance: a review. World J Diabetes. 2010:1(2):36.

22. Du T, Yuan G, Zhang M, Zhou X, Sun X, Yu X. Clinical usefulness of lipid ratios, visceral adiposity indicators, and the triglycerides and glucose index as risk markers of insulin resistance. Cardiovasc Diabetol. 2014;13(1):146

23. Marotta T, Russo BF, Ferrara LA. Triglyceride-to-HDL-cholesterol ratio and metabolic syndrome as contributors to cardiovascular risk in overweight patients. Obesity. 2010;18(8):1608-13.

24. Chen Z, Hu H, Chen M, Luo X, Yao W, Liang Q, Yang F, Wang X. Association of Triglyceride to high-density lipoprotein cholesterol ratio and incident of diabetes mellitus: a secondary retrospective analysis based on a Chinese cohort study. Lipids Health Dis. 2020;19(1):1-11.

25. Schieber LZ, Guy GP Jr, Seth P, Young R, Mattson CL, Mikosz CA, Schieber RA. Trends and patterns of geographic variation in opioid prescribing practices by state, United States, 2006-2017. JAMA Netw Open. 2019;2(3):e190665.

26. Sumner AE, Finley KB, Genovese DJ, Criqui MH, Boston RC. Fasting triglyceride and the triglyceride-HDL cholesterol ratio are not markers of insulin resistance in African Americans. Arch Intern Med. 2005;165(12):1395-400.

27. Reaven $\mathrm{G}$. Insulin resistance and coronary heart disease in nondiabetic individuals. Arterioscler Thromb Vasc Biol. 2012;32(8):1754-9.

28. Li N, Fu J, Koonen DP, Kuivenhoven JA, Snieder H, Hofker MH. Are hypertriglyceridemia and low HDL causal factors in the development of insulin resistance? Atherosclerosis. 2014;233(1):130-8.

29. Sezer $\mathrm{H}$. Insulin resistance, obesity and lipotoxicity. Obes Lipotoxicity. 2017. https://doi.org/10.1007/978-3-319-48382-5_12.

30. DeFronzo R. Insulin resistance, lipotoxicity, type 2 diabetes and atherosclerosis: the missing links. The Claude Bernard Lecture 2009. Diabetologia. 2010;53(7):1270-87.

31. Mota M, Banini BA, Cazanave SC, Sanyal AJ. Molecular mechanisms of lipotoxicity and glucotoxicity in nonalcoholic fatty liver disease. Metabolism. 2016;65(8):1049-61.

32. Barter PJ, Brewer HB Jr, Chapman MJ, Hennekens CH, Rader DJ, Tall AR. Cholesteryl ester transfer protein: a novel target for raising $\mathrm{HDL}$ and inhibiting atherosclerosis. Arterioscler Thromb Vasc Biol. 2003;23(2):160-7.

33. El Harchaoui K, van der Steeg WA, Stroes ES, Kastelein JJ. The role of CETP inhibition in dyslipidemia. Curr Atheroscler Rep. 2007;9(2):125-33.

34. Föger B, Ritsch A, Doblinger A, Wessels H, Patsch JR. Relationship of plasma cholesteryl ester transfer protein to HDL cholesterol: studies in normotriglyceridemia and moderate hypertriglyceridemia. Arterioscler Thromb Vasc Biol. 1996;16(12):1430-6.

35. Briand F, Prunet-Marcassus B, Thieblemont Q, Costard C, Muzotte E, Sordello S, Sulpice T. Raising HDL with CETP inhibitor torcetrapib improves glucose homeostasis in dyslipidemic and insulin resistant hamsters. Atherosclerosis. 2014:233(2):359-62.

36. Barter PJ, Rye K-A, Tardif J-C, Waters DD, Boekholdt SM, Breazna A, Kastelein JJ. Effect of torcetrapib on glucose, insulin, and hemoglobin A1c in subjects in the investigation of lipid level management to understand its impact in atherosclerotic events (ILLUMINATE) trial. Circulation. 2011;124(5):555-62.

37. Kahn BB, Flier JS. Obesity and insulin resistance. J Clin Investig. 2000;106(4):473-81. 
38. Ferrannini E, Natali A, Capaldo B, Lehtovirta M, Jacob S. Yki-Järvinen for the European Group for the Study of Insulin Resistance $\mathrm{H}$ : insulin resistance, hyperinsulinemia, and blood pressure: role of age and obesity. Hypertension. 1997;30(5):1144-9.

39. Patel TP, Rawal K, Bagchi AK, Akolkar G, Bernardes N, da Silva DD, Gupta $\mathrm{S}$, Singal PK. Insulin resistance: an additional risk factor in the pathogenesis of cardiovascular disease in type 2 diabetes. Heart Fail Rev. 2016;21(1):11-23.

40. Qi Q, Liang L, Doria A, Hu FB, Qi L. Genetic predisposition to dyslipidemia and type 2 diabetes risk in two prospective cohorts. Diabetes. 2012;61(3):745-52.

41. Association AD. 2. Classification and diagnosis of diabetes: standards of medical care in diabetes-2021. Diabetes Care. 2021;44(Supplement 1):S15-33.

42. Lee Y-B, Han K, Kim B, Jin S-M, Lee S-E, Jun JE, Ahn J, Kim G, Kim JH. High proportion of adult cases and prevalence of metabolic syndrome in type 1 diabetes mellitus population in Korea: a nationwide study. Diabetes Metab J. 2019;43(1):76-89.

43. Hodge AM, English DR, O'Dea K, Giles GG. Glycemic index and dietary fiber and the risk of type 2 diabetes. Diabetes Care. 2004:27(11):2701-6.

44. Willett W, Manson J, Liu S. Glycemic index, glycemic load, and risk of type 2 diabetes. Am J Clin Nutr. 2002;76(1):274S-280S.

45. Wakabayashi I, Daimon T. Comparison of discrimination for cardio-metabolic risk by different cut-off values of the ratio of triglycerides to HDL cholesterol. Lipids Health Dis. 2019;18(1):1-10.

46. Li C, Ford ES, Meng Y-X, Mokdad AH, Reaven GM. Does the association of the triglyceride to high-density lipoprotein cholesterol ratio with fasting serum insulin differ by race/ethnicity? Cardiovasc Diabetol. 2008;7(1):1-9.

47. Wakabayashi I. Influence of age and gender on triglycerides-to-HDLcholesterol ratio (TG/HDL ratio) and its association with adiposity index. Arch Gerontol Geriatr. 2012;55(3):729-34
48. Wakabayashi I. Alcohol intake and triglycerides/high-density lipoprotein cholesterol ratio in men with hypertension. Am J Hypertens. 2013;26(7):888-95.

49. Levitan EB, Cook NR, Stampfer MJ, Ridker PM, Rexrode KM, Buring JE, Manson JE, Liu S. Dietary glycemic index, dietary glycemic load, blood lipids, and C-reactive protein. Metabolism. 2008;57(3):437-43.

50. Lim T-K, Lee HS, Lee Y-J. Triglyceride to HDL-cholesterol ratio and the incidence risk of type 2 diabetes in community dwelling adults: a longitudinal 12-year analysis of the Korean Genome and Epidemiology Study. Diabetes Res Clin Pract. 2020;163:108150.

51. Kim Y-S, Han Y-E, Choi E-A, You N-Y, Lee J-W, You H-S, Kim Y, Kim J, Kang $\mathrm{H}-\mathrm{T}$. Statin use increased new-onset diabetes in hypercholesterolemic individuals: data from the Korean national health insurance servicenational health screening cohort database (NHIS-HEALS). Prim Care Diabetes. 2020;14(3):246-53.

52. Brault M, Ray J, Gomez Y-H, Mantzoros CS, Daskalopoulou SS. Statin treatment and new-onset diabetes: a review of proposed mechanisms. Metabolism. 2014;63(6):735-45.

53. Weber $P$, Raederstorff D. Triglyceride-lowering effect of omega-3 LC-polyunsaturated fatty acids-a review. Nutr Metab Cardiovasc Dis. 2000;10(1):28-37.

54. Harris WS, Bulchandani D. Why do omega-3 fatty acids lower serum triglycerides? Curr Opin Lipidol. 2006;17(4):387-93.

\section{Publisher's Note}

Springer Nature remains neutral with regard to jurisdictional claims in published maps and institutional affiliations.
Ready to submit your research? Choose BMC and benefit from:

- fast, convenient online submission

- thorough peer review by experienced researchers in your field

- rapid publication on acceptance

- support for research data, including large and complex data types

- gold Open Access which fosters wider collaboration and increased citations

- maximum visibility for your research: over 100M website views per year

At BMC, research is always in progress.

Learn more biomedcentral.com/submissions 